\title{
Smart ETA Walking Stick for Visually Impaired Person
}

\author{
Snehal N. Kolhe \\ M.E, Digital Electronics \\ Sipna COET, Amravati
}

\author{
Ajay P. Thakare, PhD \\ M.E. PhD. \\ Professor, Sipna COET, Amravati
}

\begin{abstract}
Independence is the building methodology in achieving dreams, goals and objectives in life. Visually impaired persons find themselves challenging to go out independently. There are millions of visually impaired or blind people in this world who are always in need of helping hands. For many years the white cane became a well-known attribute to blind person's navigation and later efforts have been made to improve the cane by adding remote sensor.

Blind people have big problem when they walk on the street or stairs using white cane, but they have sharp haptic sensitivity. The electronic walking stick will help the blind person by providing more convenient means of life. The main aim of this paper is to contribute our knowledge and services to the people of blind and disable society.
\end{abstract}

\section{Keywords}

Electronic Travel Aids, Infrared Sensor

\section{INTRODUCTION}

There are many guidance systems for visually impaired travelers to navigate quickly and safely against obstacles and other hazards faced. Generally, a blind user carries a white cane or a guidance dog as their mobility aid. With the advances of modern technologies many different types of devices are available to support the mobility of blind. These mobility aids are generally known as Electronic Travel Aids (ETAs). The most important function of ETA for the blind persons is to get information on the shape of the road and the position of obstacles when they are in unknown places. With this information, they need to arrive at their destinations, avoiding unexpected obstacles. The main objective of this project is to develop a simple guidance system for the blind users, using sensors, and to determine whether the blind can moves safely or not.mobility of blind. These mobility aids are generally known as Electronic Travel Aids (ETAs).

The most important function of ETA for the blind persons is to get information on the shape of the road and the position of obstacles when

\section{LITERATURE REVIEW AND RELATED WORK}

This section describes appropriate related works on the development of smart canes intended for visually impaired people. Technology can help in reducing many barriers that people with disabilities face. These kinds of technologies are referred to as Assistive Technologies (AT). There are many types of disabilities, including physical disabilities, hearing impaired, and visually-impaired. AT has been utilized in assisting them. However, developing an AT is expensive, making their selling price high. A few of them are enumerated below which is a humanitarian mission to help the blind children by training them to utilize their brains to learn a set of objects around them. Another system utilizes two sets of ultrasonic sensors to detect obstacles in front and below the stick. These sensors use Pulse Width Modulation to change the vibration pattern for differing range of obstacles. A smart walking stick developed by researches in Coimbatore use various sensors for obstacles, pot holes, and moisture detection viz. Ultrasonic sensor, Pit sensor, and Moisture sensors respectively. The stick fails to detect dynamic obstacles not directly in the range of the ultrasonic sensor

\section{Details of topic}

Many ETAs and robot technologies have been applied, to guide the blinds that aimed at improving their mobility in terms of safety, to detect obstacles on the ground, uneven surfaces, holes, steps, and puddles.

\section{A. C-5 Laser Cane}

It was introduced in 1973 by Benjamin. It is based on optical triangulation with three laser diodes and three photo-diodes as receivers. The Laser Cane can detect obstacles at head-height, drop-offs in front of the user, and obstacles up to a range of 1.5 $\mathrm{m}$ or $3.5 \mathrm{~m}$ ahead of the user.

\section{B. Sonic Torch}

It is a battery operated hand held device basically operates by transmitting the ultrasound in the forward direction and receiving the reflected sound beam from the nearest object.

\section{Mowat Sensor}

It is a commercially available hand-held ultrasonic-based device that informs the user of the distance to detected objects by means of tactile vibrations. The frequency of the vibration is inversely proportional to the distance between the sensor and the object.

\section{Sonic Path Finder}

It alarms the blind when detecting the obstacle by the acoustic difference. However, it does not provide the accurate path and the position of an obstacle. It alarms the blind when detecting the obstacle by the acoustic difference. However, it does not provide the accurate path and the position of an obstacle.

\section{E. Meldog}

It uses the artificial intelligence, unlike in the sonic pathfinder. It can provide the accurate position of an obstacle using the ultrasound and laser sensors. But, in general, it is relatively large and heavy.

\section{F. Navbelt (1989)}

It is a portable device equipped with ultrasonic sensors and a computer . It produced a $120^{\circ}$-wide view of the obstacles ahead of the user (similar to a radar screen image). This image was then translated into a series of directional (stereophonic) audio cues through which the user could determine which directions were blocked by obstacles.

\section{System Description}

Fig. 1 shows a blind user walking with an electronic stick. Two ultrasonic sensors are mounted on the stick having range from $20-350 \mathrm{cms}$ (set to different ranges). Two Infrared sensors are also implemented on the lower side of stick for avoiding small obstacles ranging from $2-10 \mathrm{cms}$. A switch that can be operated with the thumb (in worst condition) that allows the blind user 
to send a general message (I am in trouble, help me) on a saved mobile no. for help. Vibrating sensors along with a buzzer used for beep and vibration if stick is about to hit with any obstacle. Circuit box contain combination of GSM300/900 module and microcontroller circuitry. The co-operation between the Ultrasonic and IR sensors are utilized to create a complementary system that is able to give reliable distance measurement.

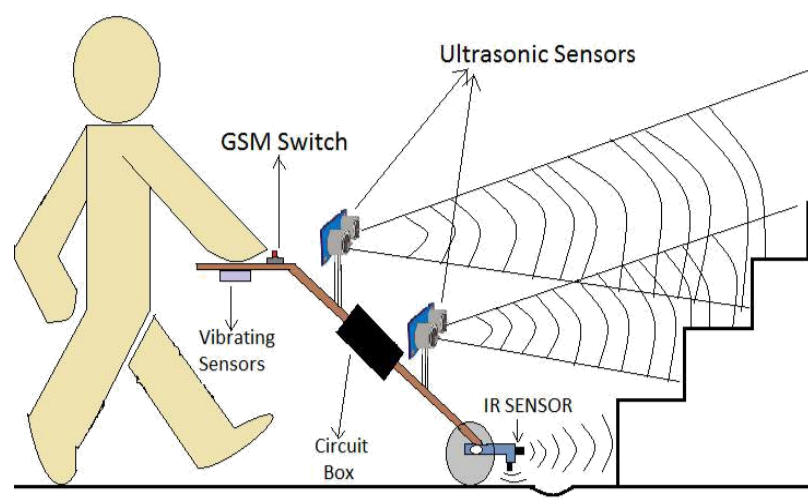

Table: Output Signal Algorithm For Stick

TABLE I OUTPUT SIGNAL ALGORITHM FOR STICK

\begin{tabular}{|c|c|c|}
\hline $\begin{array}{l}\text { Distance (from } \\
\text { the stick in } \mathrm{cms} \text { ) }\end{array}$ & Time (sec) & Type of signal \\
\hline 350 & 2.0 & \multirow{2}{*}{$\begin{array}{c}\text { Very Slow } \\
\text { Beeps }\end{array}$} \\
\hline 300 & 1.8 & \\
\hline 250 & 1.5 & \multirow{2}{*}{ Slow Beeps } \\
\hline 200 & 1.4 & \\
\hline 150 & 1.0 & \multirow{3}{*}{$\begin{array}{c}\text { Beeps + } \\
\text { Vibrations }\end{array}$} \\
\hline 130 & 0.9 & \\
\hline 100 & 0.8 & \\
\hline 80 & 0.5 & \multirow{2}{*}{$\begin{array}{l}\text { Fast Beeps } \\
\text { +Vibrations }\end{array}$} \\
\hline 60 & 0.3 & \\
\hline $40-50$ & 0.1 & \multirow{2}{*}{$\begin{array}{c}\text { Very Fast Beep + } \\
\text { Vibrations }\end{array}$} \\
\hline $20-30$ & 0.05 & \\
\hline $\begin{array}{c}\text { 5-10 (due to } \\
\text { Infrared Sensor) }\end{array}$ & - & $\begin{array}{l}\text { Continuous } \\
\text { Vibrations }\end{array}$ \\
\hline
\end{tabular}

\section{The Features Are}

Different types of vibrations for different obstacles. Automatic (during night time i.e. LDR dependent) high intensity (Red color) LEDs. A small Torch on the top. A user controlled horn for traffic. More efficient and inexpensive than other devices. Easy to access for blinds. Code can be upgrade or change easily.

\section{Proposed System}

The proposed system aims to enable the visually challenged to navigate through a course of dynamic objects.

\section{Ultrasonic Sensor}

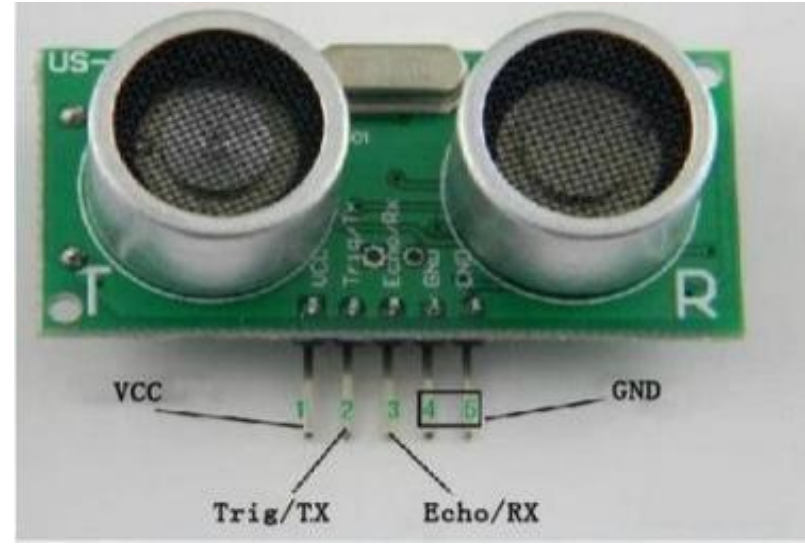

Fig. 1 Ultrasonic Sensor

Ultrasonic Sensor (transducers) is a type of sensor that uses sound waves to detect an object or target .It works on similar principle of radar or sonar which generates high frequency sound waves and evaluates the echo which is received back by the sensor. Sensors calculate the time interval between sending the signal and receiving the echo to determine the distance to an object.

\section{Infrared Sensor}

An IR sensor is an electronic device that emits and/or detects infrared radiations in order to sense some aspect of its surroundings. When an object is close to the sensor, the light from the IR transmitter led bounces off the object and received by receiver led.

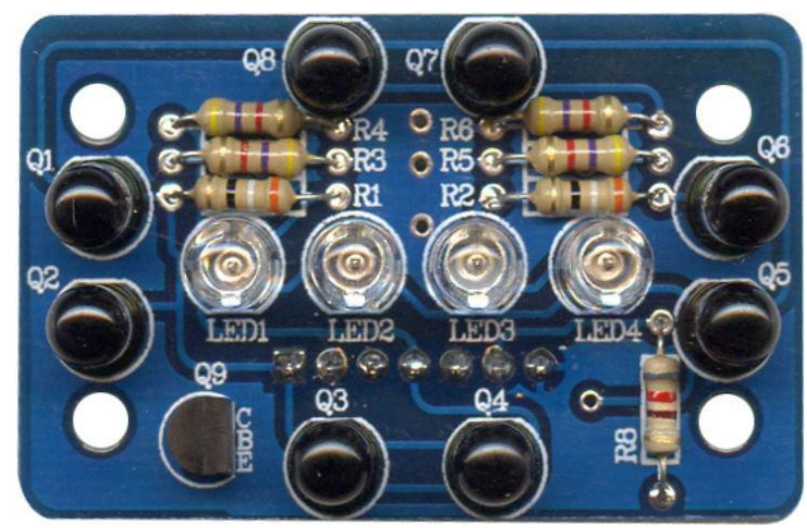

Fig. 2. Infrared Sensor

\section{GSM}

GSM stands for Global System for Mobile Communication and is an open, digital cellular technology used for transmitting mobile voice and data services. GSM module makes use of narrowband Time Division Multiple Access (TDMA) technique for transmitting signals 


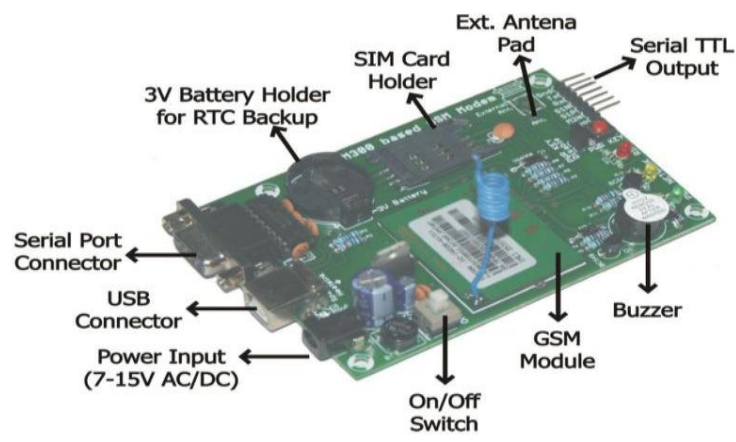

Fig.3 GSM Module

\section{ATmega328}

ATmega328 is a microcontroller, can be also use in arduino board which is an open-source physical computing platform based on Atmel microcontrollers, and a development environment for writing software for the board. It can be used to develop interactive objects, taking inputs from a variety of switches or sensors, and controlling other physical outputs

\section{ATMega328 Pinout Diagram}

\begin{tabular}{|c|c|c|c|}
\hline PC6 & 1 & 28 & $\checkmark \mathrm{PC} 5$ \\
\hline PDO & 2 & 27 & $\checkmark \mathrm{PC} 4$ \\
\hline PD1 & 3 & 26 & $\checkmark \mathrm{PC} 3$ \\
\hline PD2 & 4 & 25 & $\neg \mathrm{PC} 2$ \\
\hline PD3 & 5 & 24 & $\checkmark \mathrm{PC} 1$ \\
\hline PD4 & 6 & 23 & $\checkmark \mathrm{PCO}$ \\
\hline $\mathrm{vec}$ & 7 & 22 & GND \\
\hline GND & 8 & 21 & $\square$ ARE \\
\hline PB6 & 9 & 20 & $\mathrm{AVCC}$ \\
\hline PB7 & 10 & 19 & PB5 \\
\hline PD5 & 11 & 18 & $\neg$ PB4 \\
\hline PD6 & 12 & 17 & PB3 \\
\hline PD7 & 13 & 16 & PB2 \\
\hline $\mathrm{PBO}$ & 14 & 15 & PB1 \\
\hline
\end{tabular}

\section{FUNCTIONAL DESCRIPTION}

\section{A. Ultrasonic Sensor}

Ultrasound waves or ultrasonic waves are the terms used to describe elastic waves with frequency greater than $20,000 \mathrm{~Hz}$ and normally exist in solids, liquids, and gases. An ultrasonic wave

moves at a velocity (the wave velocity) that is determined by the material properties and shape of the medium Laplace proposed that the speed of a longitudinal wave is given by

Where, from equation (1), $\mathrm{P}$ is the pressure, is density and $\gamma$ is dimensionless constant. At atmospheric pressure $\gamma=1.4, \mathrm{P}=$ $0.76 \times 13600 \times 9.8 \mathrm{~Pa}$ and $=1.29$, therefore, $=332 \mathrm{~m} / \mathrm{s}$, it means ultrasonic waves travel in air with speed of $332 \mathrm{~m} / \mathrm{s}$. The function of the transducers is to convert electrical energy into mechanical energy which directly corresponds to ultrasonic vibration .

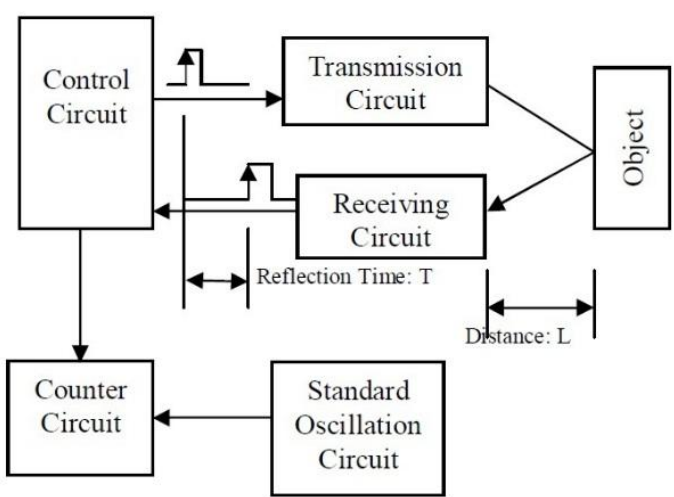

Fig 4 Block Diagram of distance calculator

figure shows the principles of measuring distance and is called the "pulse reflection method" which count the number of reference pulses [10]. This method is used to measure reflection time up to the object between transmitting pulse and receiving pulse of the ultrasonic wave. The relationship between the distance up to the object $\mathrm{L}$ and the reflecting time $\mathrm{T}$ can be expressed by

$$
\mathrm{L}=\mathrm{c} \times \mathrm{T} / 2
$$

Where, $\mathrm{c}$ is the velocity of wave.

Table II Results of Ultrasonic Sensor up to $200 \mathrm{c}$

\begin{tabular}{|c|c|c|c|}
\hline $\begin{array}{c}\text { Range } \\
\text { (inches) }\end{array}$ & $\begin{array}{c}\text { Calculated } \\
(\mathbf{m V})\end{array}$ & $\begin{array}{c}\text { Measured } \\
(\mathbf{m V})\end{array}$ & Error \% \\
\hline 0 & 0 & 0 & - \\
\hline 10 & 100 & 95 & 5 \\
\hline 20 & 200 & 191 & 4.5 \\
\hline 30 & 300 & 295 & 1.67 \\
\hline 40 & 400 & 393 & 1.75 \\
\hline 50 & 500 & 497 & 0.6 \\
\hline 60 & 600 & 596 & 0.67 \\
\hline 70 & 700 & 697 & 0.428 \\
\hline 80 & 800 & 794 & 0.75 \\
\hline
\end{tabular}

The table shows the error \% of gathered data of SR04, as shown in fig. 2, ultrasonic sensor which provides approximately $2 \mathrm{~cm}-300 \mathrm{~cm}$ non-contact measurement function with accuracy of $3 \mathrm{~mm}$. Fig. 6 shows the working flow chart of an ultrasonic sensor. 


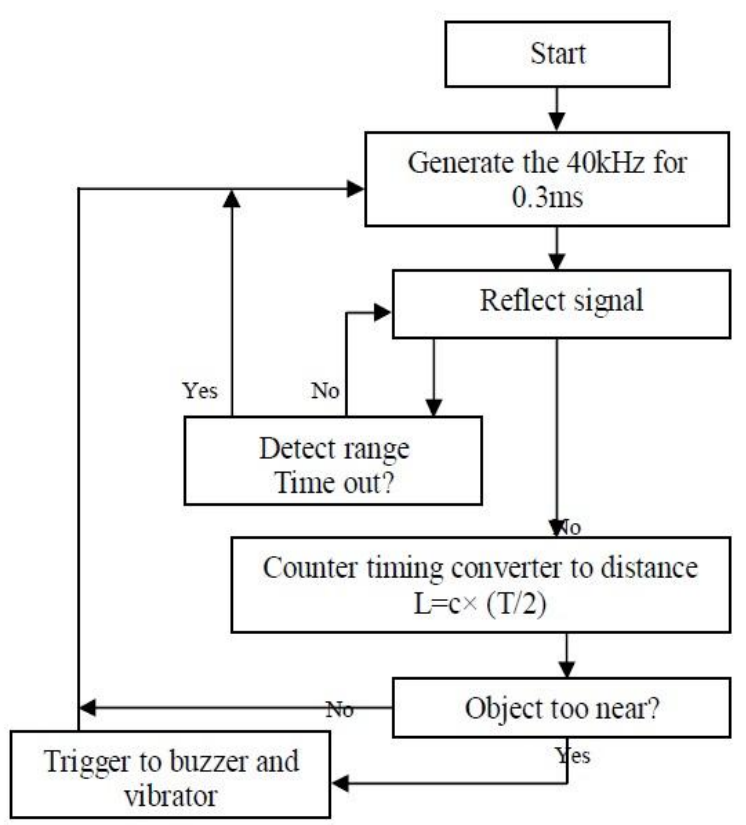

Fig.6 Flow Chart of Ultrasonic Sensor

Fig. 6 shows the principles of measuring distance and is called the "pulse reflection method" which count the number of reference pulses. This method is used to measure reflection time up to the object between transmitting pulse and receiving pulse of the ultrasonic wave. The relationship between the distance up to the object $\mathrm{L}$ and the reflecting time $\mathrm{T}$ can be expressed by

$\mathrm{L}=\mathrm{c} \times \mathrm{T} / 2$

Where, $\mathrm{c}$ is the velocity of wave.

The table shows the error \% of gathered data of SR04, as shown in fig. 2, ultrasonic sensor which provides approximately $2 \mathrm{~cm}-300 \mathrm{~cm}$ non-contact measurement function with accuracy of $3 \mathrm{~mm}$. Fig. 6 shows the working flow chart of an ultrasonic sensor.

\section{Infrared Sensor}

In an unknown environment, it is important to know about the nature of surface properties in order to interpret IR sensor output as a distance measurement. IR sensors using reflected light intensity to estimate the distance from an object. The function of the detector led $\left(\mathrm{R}_{\mathrm{X}}\right)$ is to detect the energy reflected by an obstacle from the emitter IR led $\left(\mathrm{T}_{\mathrm{X}}\right)$. The signal returned from the sensor is dependent on the energy emitted from the transmitter LED

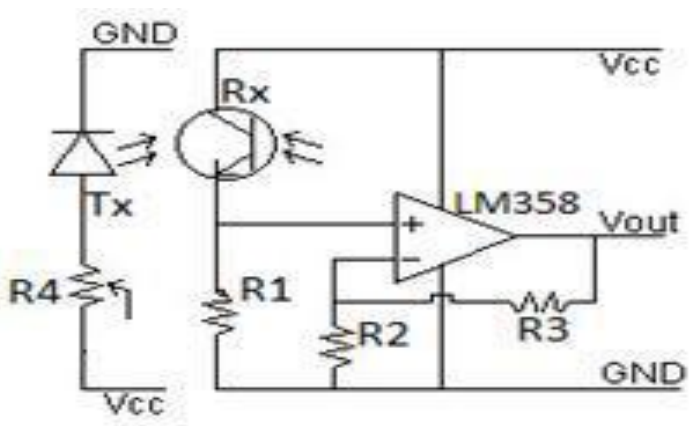

Fig. 7 Circuit diagram of comparator
Fig. 7 shows a comparator op-amp IC LM358 which compares a continuous signal voltage applied at positive input of opamp with a known reference voltage at the other input

$\mathrm{VO}=(1+\mathrm{R} 3 / \mathrm{R} 2) \times \mathrm{VIN}$

Where VIN is continuous signal voltage.

Gain of op-amp $=(1+\mathrm{R} 3 / \mathrm{R} 2)$

where, R2, R3 determine the amplification of op-amp.

A ratio can be determined by measuring the voltageentering the op-amp and knowing the threshold value needed atVO.

Considering an

approximate gain of op-amp $\approx 3$ and assuming $\mathrm{R} 3=10 \mathrm{k} \Omega$, R 2 $=4.7 \mathrm{k} \Omega$. Resistance $\mathrm{R} 1=220 \mathrm{k} \Omega$ must be

larger than maximum resistance of detector led. R4 $(\sim 150 \Omega)$ is a voltage divider, calibrate according to intensity of emitter led ,When the intensity of emitter led is high, more energy will fall on detector led and resistance of detector is low, so the value of the potential (VIN) is high. Similarly when the intensity is low, the resistance of the detector is high and so the value of potential is low. This potential is compared with a reference potential. According to these compared potentials the output will be 1 or 0 i.e. 'ON' or' OFF'.

\section{GSM}

A GSM (Global System for Mobile communication) module is a specialized type of module which accepts a SIM (Subscriber Identity Module) card, and operates over a subscription to a mobile operator. When a GSM module is connected to a microcontroller (ATmega328), this allows the microcontroller to use the GSM module to communicate over the mobile network. A GSM module exposes an interface that allows applications to send and receive messages over the module interface. To perform these tasks, a GSM module must support an "extended AT command set" for sending/receiving SMS messages. The AT commands are sent by the microcontroller to the module. The module sends back an Information Response i.e. the information requested by the action initiated by the AT command. This is followed by a Result Code. The result code tells about the successful execution of that command. Text message may be sent through the module by interfacing only three signals of the serial interface of module with microcontroller i.e., $T_{X} D, R_{X} D$ and GND. In this scheme RTS and CTS signals of serial port interface of GSM Modem are connected with each other.

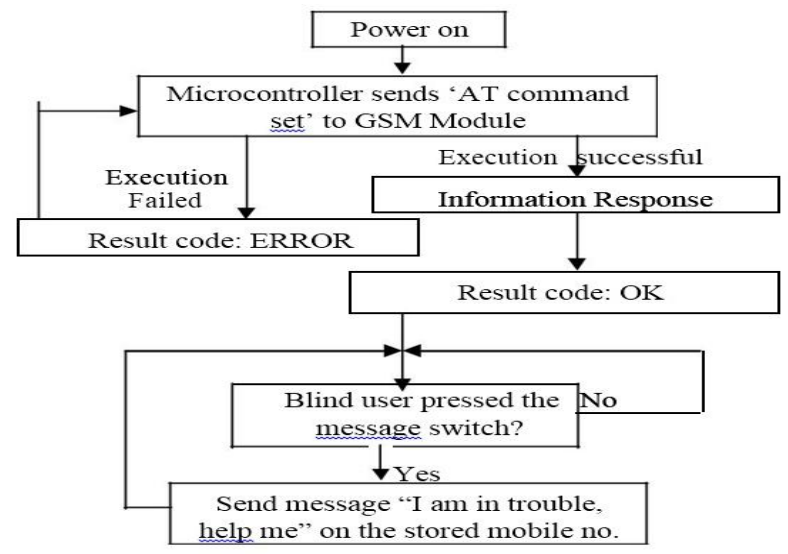

Fig.8 Flow chart of GSM module 
$\mathrm{AT}+\mathrm{CMGF}=1$, this command configures the GSM module in text mode. $\mathrm{AT}+\mathrm{CMGS}=+917708377615$, this command sends the mobile number of the recipient mobile to the GSM module. GSM configured with microcontroller using two modesautomatic and manual. If the object is too near and system is sending alert sounds and vibrations then automatically it send the message to the stored mobile number. Fig. 8 shows an algorithmic flow chart of GSM module for blind stick

\section{Microcontroller (ATmega328)}

An ATmega328, has 14 digital input/ output pins (of which 6 can be used as PWM (Pulse Width Modulation) outputs), six analog inputs with one $16 \mathrm{MHz}$ crystal oscillator. For Communication purposes it has a number of facilities for communicating with a computer, or with other microcontrollers. The ATmega328 provides UART TTL serial communication which is available on digital pins $0(\mathrm{RX})$ and 1(TX).

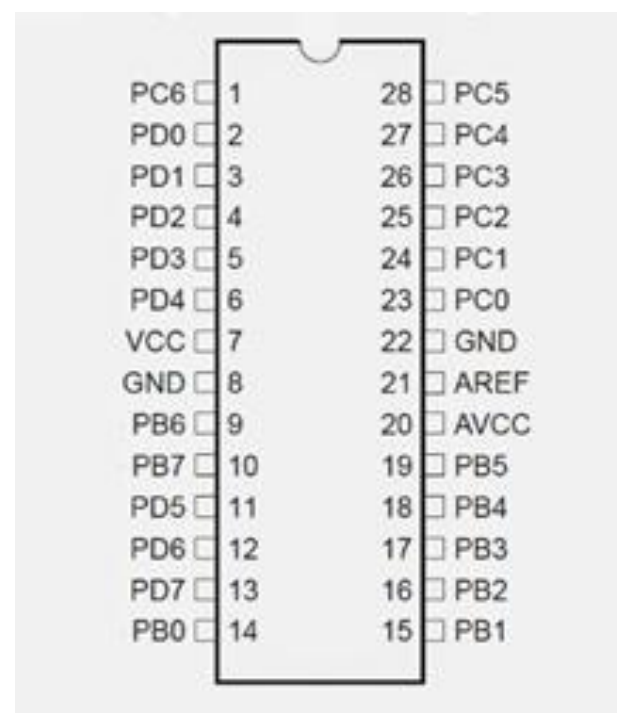

Fig. ATMega 328 Pinout Diagram

\section{CONCLUSION}

This system can be applied in the straight path, right angle path and the curved path. At least $1 \mathrm{~m}$ width is required for the proper management of the stick. The broad beam angle ultrasonic sensors enable wide range obstacle information. Major drawback of infrared sensors is their non-linear response i.e. a big change in output voltage does not always indicate a big change in range. The main functions of this system are the clear path indication and the environment recognition. With the help of electronic walking stick blind, people can improve more than $15-20 \%$ travel speed, reduce minor collision, do not lose their way, and increase safety as compare to unaided equipment's. Future work includes installation of GPS system along with additional sensors like accelerometers, PIR motion detector and digital compass which tell the exact location of the user.

\section{REFERENCES}

[1] Manoj Badoni and Sunil Semwal, "Discrete Distance And Water Pit Indicator Using Avr Atmega8 In Electronic Travel Aid For Blind", International Journal of Disaster Recovery and Business Continuity Vol. 2, November, 2011.

[2] Sung Jae Kang, Young Ho, Kim, In Hyuk Moon, "Development Of An Intelligent Guide-Stick For The Blind", IEEE International Conference on Robotics \& Automation Seoul, Korea, May 21-26, 2001.

[3] Alessio Carullo and Marco Parvis, "An Ultrasonic Sensor For Distance Measurement In Automotive Applications", IEEE Sensors Journal, Vol.1, No.2, August 2001.

[4] http://www.societyofrobots.com/schematics_infraredemitd et.shtml

[5] Zul Azizi Hailani, Sakinah Jamaludin, "An Electronically Guided Walking Stick For The Blind" University Tenaga Nasional, Malaysia.

[6] Johann Borenstein and Iwan Ulrich, "The Guide Cane- A Computerized Travel Aid for The Active Guidance Of Blind Pedestrians", IEEE International Conference on Robotics and Automation, Albuquerque, NM, Apr. 21-27, 1997. 